\title{
Acute cholangitis in intensive care units: clinical, biological, microbiological spectrum and risk factors for mortality: a multicenter study
}

Jean-Rémi Lavillegrand ${ }^{1,2,13}$, Emmanuelle Mercier-Des-Rochettes ${ }^{3}$, Elodie Baron ${ }^{4}$, Frédéric Pène ${ }^{4}$, Damien Contou ${ }^{5}$, Raphael Favory ${ }^{6}$, Sébastien Préau ${ }^{6}$, Arnaud Galbois ${ }^{7}$, Chloé Molliere ${ }^{7}$, Arnaud-Félix Miailhe ${ }^{8}$, Jean Reignier ${ }^{8}$, Mehran Monchi ${ }^{3}$, Claire Pichereau ${ }^{9}$, Sara Thietart ${ }^{1}$, Thibault Vieille ${ }^{10}$, Gael Piton ${ }^{10}$, Gabriel Preda ${ }^{11}$, Idriss Abdallah ${ }^{12}$, Marine Camus ${ }^{2,14}$, Eric Maury ${ }^{1,2}$, Bertrand Guidet ${ }^{1,2}$, Guillaume Dumas ${ }^{1,2}$ and Hafid Ait-Oufella ${ }^{1,2,13^{*}}$ (1)

\begin{abstract}
Background: Little is known on the outcome and risk factors for mortality of patients admitted in Intensive Care units (ICUs) for Acute cholangitis (AC).

Methods: Retrospective multicenter study included adults admitted in eleven intensive care units for a proven AC from 2005 to 2018. Risk factors for in-hospital mortality were identified using multivariate analysis.

Results: Overall, 382 patients were included, in-hospital mortality was 29\%. SOFA score at admission was 8 [5-11]. Biliary obstruction was mainly related to gallstone (53\%) and cancer (22\%). Median total bilirubin and PCT were respectively $83 \mu \mathrm{mol} / \mathrm{L}$ [50-147] and $19.1 \mu \mathrm{g} / \mathrm{L}$ [5.3-54.8]. Sixty-three percent of patients $(n=252)$ had positive blood culture, mainly Gram-negative bacilli (86\%) and $14 \%$ produced extended spectrum beta lactamase bacteria. At ICU admission, persisting obstruction was frequent (79\%) and biliary decompression was performed using therapeutic endoscopic retrograde cholangiopancreatography (76\%) and percutaneous transhepatic biliary drainage (21\%). Adjusted mortality significantly decreased overtime, adjusted OR for mortality per year was $0.72[0.54-0.96](p=0.02)$. In a multivariate analysis, factors at admission associated with in-hospital mortality were: SOFA score (OR 1.14 [95\% Cl 1.05-1.24] by point, $p=0.001$ ), lactate (OR 1.21 [95\% Cl 1.08-1.36], by $1 \mathrm{mmol} / \mathrm{L}, p<0.001)$, total serum bilirubin (OR 1.26 [95\% Cl 1.12-1.41], by $50 \mu \mathrm{mol} / \mathrm{L}, p<0.001)$, obstruction non-related to gallstones $(p<0.05)$ and AC complications (OR 2.74 [95\% Cl 1.45-5.17], $p=0.002$ ). Time between ICU admission and biliary decompression $>48 \mathrm{~h}$ was associated with in-hospital mortality (adjusted OR 2.73 [95\% Cl 1.30-6.22], $p=0.02$ ).
\end{abstract}

Conclusions: In this large retrospective multicenter study, we found that AC-associated mortality significantly decreased overtime. Severity of organ failure, cause of obstruction and local complications of AC are risk factors for mortality, as well as delayed biliary drainage $>48 \mathrm{~h}$.

\footnotetext{
*Correspondence: hafid.aitoufella@aphp.fr

${ }^{1}$ Assistance Publique - Hôpitaux de Paris (AP-HP), Service de médecine

intensive et réanimation, Hôpital Saint-Antoine, 184 rue du Faubourg Saint-Antoine, 75571 Paris Cedex 12, France

Full list of author information is available at the end of the article
} permits use, sharing, adaptation, distribution and reproduction in any medium or format, as long as you give appropriate credit to the original author(s) and the source, provide a link to the Creative Commons licence, and indicate if changes were made. The images or other third party material in this article are included in the article's Creative Commons licence, unless indicated otherwise in a credit line to the material. If material is not included in the article's Creative Commons licence and your intended use is not permitted by statutory regulation or exceeds the permitted use, you will need to obtain permission directly from the copyright holder. To view a copy of this licence, visit http://creativecommons.org/licenses/by/4.0/. The Creative Commons Public Domain Dedication waiver (http://creativeco mmons.org/publicdomain/zero/1.0/) applies to the data made available in this article, unless otherwise stated in a credit line to the data. 
Keywords: Cholangitis, Outcome, Prognosis, Microbiology, Intensive care unit

\section{Introduction}

Acute cholangitis (AC), also called ascending cholangitis, is a bacterial infection of the biliary system, due to partial or complete obstruction of the bile duct or hepatic ducts. The most common cause of biliary obstruction is gallstone or malignant stricture, but obstruction caused by primary sclerosing cholangitis, amyloid depositions or parasite biliary invasions can also be observed. Classically, patients with AC present with persisting high fever for more than $24 \mathrm{~h}$, right upper quadrant abdominal pain and jaundice. The International Consensus Meeting held in Tokyo in 2006 proposed to use inflammatory response biomarkers, liver function tests and imaging in order to optimize diagnosis, localize biliary obstruction and guide the etiological diagnosis [1].

$\mathrm{AC}$ diagnosis and management should be considered as an emergency because it may be responsible for lifethreatening organ failure and death. Intensive Care unit (ICU) admission of patients with AC is not rare, as it is responsible for $5 \%$ of septic shocks [2] and for 10 to $29 \%$ of intra-abdominal sepsis [3-5]. The cornerstone of the management of AC is based on fluid resuscitation, antibiotics, organ support therapy, and biliary drainage. However, not all ICUs have access to endoscopic retrograde cholangiopancreatography (ERCP), interventional radiology or hepatobiliary surgery at night or on week-end.

Our study aimed to describe over a 14-year period the demographical, clinical and microbial patterns of critically ill patients admitted in ICUs for AC, to describe therapeutic management and analyze risk factors for in-hospital mortality, including time to biliary decompression.

\section{Material and methods Study design}

Between January 2005 and March 2018, all adult patients ( $\geq 18$ years of age) admitted in 11 French ICUs (cf. Additional files 1 and 2) for acute cholangitis were identified by searching hospital databases for codes K803 or K830 as a principal or associated diagnosis according to the International Classification of Diseases $\left(10^{\text {th }}\right.$ version). Acute cholangitis was defined by the association of fever, jaundice, imaging abnormalities and/or biliary obstruction, according to the International Guidelines $[6,7]$.

\section{Data collection}

Data were collected retrospectively by local investigators using electronic case report forms (eCRF) then centralized and anonymized. The following data were collected: age, gender, cardiovascular diseases (coronary artery diseases or chronic heart failure), hypertension, other comorbidities (e.g. diabetes mellitus, cancer, immune deficiency, cirrhosis, chronic renal failure), clinical symptoms (abdominal pain, jaundice, diarrhea, chills) at ICU admission. Disease severity was evaluated using the Simplified Acute Physiology Score (SAPS II), the Sequential Organ Failure Assessment (SOFA) score, and the use of organ support therapy.

Specific AC-related parameters occurring during ICU stay were also recorded: presence of liver abscesses; parameters related to biliary drainage (method, time from admission), ERCP complications, microbial analysis, anti-microbial therapy. Non-optimal biliary drainage was defined as persistent partial biliary obstruction after the first decompression procedure. Sepsis and septic shock were defined using the Third International Consensus Definitions for Sepsis and Septic Shock (Sepsis-3) [8]. Anti-microbial therapy was considered as appropriate if the first line therapy was active on the isolated pathogen (blood and/or biliary cultures) according to the EUCAST guidelines [9].

All authors had access to the study data and reviewed and approved the final manuscript.

Quality was assessed according to the STROBE checklist.

\section{Statistical analysis}

Continuous variables were described as median [interquartile ranges (IQRs)] and categorical variables as proportions. Comparisons were made using the Fisher's exact test or Wilcoxon rank-sum test when appropriate. First, factors associated with in-hospital mortality (model 1) were assessed using a multivariate mixed logistic model, considering the center as a random variable. Covariates associated with outcome at the 0.2 level in univariate analysis were selected upon clinical relevance and were included in the model as fixed effect. A multiple backward stepwise selection procedure eliminated those variables with an exit threshold set at $p=0.05$, after testing for collinearity between variables and checking the assumption of log-linearity as described above.

Second, to investigate the prognostic significance of time to biliary drainage on in-hospital mortality (model 2 ), the analysis took death into account as a competing risk of drainage. Thus, we used a Landmark analysis restrained to patients still alive 3 days after ICU admission. This landmark time was fixed before the analysis, because it was considered as the maximum time to 
obtain an available technical center. Then the time to decompression was assessed with multivariate mixed model with center as a random variable and adjusted on SOFA score (day 3), obstacle nature, local complications of cholangitis, prothrombin time and appropriate empirical antibiotics.

To handle missing values as potential confounders, multiple imputation with chained equation was used. Primary analyses were performed on the complete cases, assuming missing completely at random covariates. Then, sensitivity analyses for such assumptions were performed, based on multiple imputation with chained equation.

To test for the significance of the center effects, we used permutations test, a recommended approach to test for random effect (for details [10]).

Odd-Ratios (OR) in the two final models are given with their 95\% confidence intervals (CI). All tests were two-sided and p-values lower than 5\% were considered to indicate significant associations. Analyses were performed using R statistical platform, version 3.0.2 (https:// cran.r-project.org/).

\section{Results}

\section{General characteristics}

Over a 14-year period, 382 patients were included in 11 ICUs $(5$ tertiary hospitals, 5 general hospitals and 1 private hospital) in France (Additional files 1 and 2). Median inclusion number per center was 28 [18-48]. The median age was $72[62-81]$ years with a twofold higher incidence of men (63\%). Patient characteristics are summarized in Table 1 . Comorbidities were frequent, notably diabetes $(n=112,30 \%)$, cardiovascular diseases $(n=301,79 \%)$ and active solid tumors $(n=118,31 \%)$. Abdominal pain $(73 \%)$ and jaundice (63\%), which are classical clinical signs of $\mathrm{AC}$, were frequently observed but fever (defined by a temperature $>38{ }^{\circ} \mathrm{C}$ ) was only reported in 116 patients (31\%). The most commonly used imaging method for diagnosis of AC was abdominal CT-scan (167/382; 46\%), and less frequently ultrasonography $(65 / 382 ; 18 \%)$. As expected, the 2 main causes of biliary obstruction were gallstones (201/382 patients; $53 \%)$ and tumors (85/382 patients; $22 \%)$. AC due to primary sclerosing cholangitis was rare $(15 / 382,4 \%)$.

At ICU admission, patients with AC had severe diseases, with sepsis (370/382; 97\%), septic shock (170/382; $44 \%)$ and a high SOFA score (8 [5-11]) [8]. They needed frequent organ support therapies, including mechanical ventilation (38\%), vasopressor infusion (61\%) and renalreplacement therapy (26\%) (Table 1). A high proportion of patients had renal failure, coagulopathy and lactic acidosis (Table 2). Finally, in-ICU mortality reached $21 \%$, half of the deaths occurring within the first 10 days
Table 1 General characteristics of the 382 patients included

\begin{tabular}{ll}
\hline Parameters & $\boldsymbol{n}(\%)$ or Med. [IQR] \\
\hline Age & $72[62-81]$ \\
BMI & $26[23-81]$ \\
Male gender & $242(63)$ \\
SAPS2 & $50[38-64]$ \\
SOFA at admission & $8[5-11]$ \\
Comorbidities & \\
Diabetes & $116(30)$ \\
Hypertension & $235(62)$ \\
Cardiovascular disease & $301(79)$ \\
Chronic kidney disease & $46(12)$ \\
Cirrhosis & $20(5)$ \\
Cancer and hematologic malignancies & $141(37)$ \\
Organ support therapy during ICU stay & \\
Invasive mechanical ventilation & $144(38)$ \\
Invasive mechanical ventilation duration: days & $5[2-10]$ \\
Renal replacement therapy & $91(26)$ \\
Vasopressor infusion & $222(61)$ \\
Vasopressor infusion duration: hours & $48[24-91]$ \\
Persisting obstruction at ICU admission & $298(79)$ \\
Biliary drainage procedure & \\
Percutaneous derivation & $78(21)$ \\
ERCP & $285(76)$ \\
Surgery & $28(7)$ \\
Complications before ERCP & \\
Associated pancreatitis & $94(25)$ \\
Liver abscess & $31(8)$ \\
Complications after ERCP & \\
Bleeding & $22(9)$ \\
Gastrointestinal perforation & $7(3)$ \\
Pancreatitis & $10(4)$ \\
Death in ICU & $105(29)$ \\
Death in hospital & \\
Length of stay in ICU: days & \\
Length of stay in hospital: days & \\
\hline SAPS2, & \\
\hline
\end{tabular}

SAPS2, simplified acute physiology score; SOFA, Sepsis-related organ failure; $E R C P$, endoscopic retrograde cholangiopancreatography

of ICU admission. Time between ICU admission and appropriate antibiotic therapy administration was $0[-4$; 3] hours.

\section{Biliary drainage}

Overall, biliary drainage was performed in $86 \%$ $(n=330 / 382)$ of the patients either before ICU admission $(n=32 / 330)$ or during ICU stay $(n=298 / 330)$. The median time to biliary drainage was 24 [9-48] hours. Endoscopic Retrograde Cholangiopancreatography (ERCP) was the first-line technique used for biliary 
Table 2 Clinical and biological parameters at ICU admission

\begin{tabular}{ll}
\hline Parameters & $\boldsymbol{n}(\%)$ or Med. [IQR] \\
\hline Clinical signs & \\
Abdominal pain & $278(73)$ \\
Jaundice & $240(63)$ \\
Chills & $118(31)$ \\
Temperature & $37.4[36.5-38.3]$ \\
Temperature $>38.0{ }^{\circ} \mathrm{C}$ & $116(31)$ \\
Biological parameters & \\
Leucocytes (Giga/L) & $15.29[10.42-21.62]$ \\
Hemoglobin (g/dL) & $11.1[9.6-12.9]$ \\
Platelets (Giga/L) & $160.0[98.2-240.0]$ \\
Blood alanine aminotransferase (X ULN) & $4.1[2-8.2]$ \\
Blood aspartate aminotransferase (X ULN) & $3.5[1.6-7.2]$ \\
Total blood bilirubin ( $\mu$ mol/L) & $83[50-147]$ \\
Lipase (IU/L) & $55[18-276]$ \\
Serum creatinine $(\mu m o l / L)$ & $143.5[81-232]$ \\
Prothrombin time $<50 \%$ & $125(34)$ \\
APTT & $1.2[1-1.45]$ \\
Factor $V<50 \%$ & $30(11)$ \\
C-reactive protein $(\mathrm{mg} / \mathrm{L})$ & $144.5[75.25-233]$ \\
Procalcitonin $(\mathrm{ng} / \mathrm{mL})$ & $19.11[5.27-54.81]$ \\
Arterial lactate $(\mathrm{mmol} / \mathrm{L})$ & $2.61[1.5-4.6]$ \\
\hline APTT, &
\end{tabular}

APTT, activated partial thromboplastin time; X ULN, X times upper limit normal

drainage in 285 patients $(N=24$ before ICU admission), associated with stent placement in $48 \%$ of the interventions $(n=137 / 285)$. Completion rate of ERCP within 24 or $48 \mathrm{~h}$ did not change during the 2005-2018 period. The procedure was described as optimal in $75 \%$ of the cases by the physician who performed the drainage. Non-optimal procedure was more frequently reported in non-gallstone related $\mathrm{AC}$, when compared with gallstone related AC $(11 / 38$ versus $15 / 201 ; p=0.006)$. The median time between ICU admission and biliary drainage was 39.5 [17.3-84.8] hours. Percutaneous biliary derivation was performed in 78 patients $(21 \%)$ and surgical decompression was rarely done $(n=28,7 \%)$.

Following ERCP procedure, 40 complications (14\%) were reported including bleeding requiring red blood cell transfusion $(n=22,9 \%)$; pancreatitis $(n=10,4 \%)$ and gastro-intestinal perforation $(n=7,3 \%)$.

\section{Microbiologic analysis}

Positive blood culture was frequently reported in patients with AC $(n=252 / 373,68 \%)$. Bile culture was only performed in $189 / 375$ patients and was positive in $79 \%$ of the cases $(149 / 189)$. When blood and bile were simultaneously collected $(n=189)$, both culture samples were positive in $56 \%$ of the cases $(n=106 / 189)$. Microbiological
Table 3 Microbiological data in blood and bile cultures

\begin{tabular}{lll}
\hline Germs & Blood & Bile \\
\hline Samples performed & 373 & 189 \\
Positive culture & $252(68)$ & $149(79)$ \\
$>1$ pathogen/culture & $91(36)$ & $75(40)$ \\
Total pathogens & 379 & 261 \\
Gram-negative bacilli & $282(74)$ & $155(59)$ \\
Escherichia coli & $162(43)$ & $79(30)$ \\
Klebsiella pneumoniae & $39(10)$ & $18(7)$ \\
Enterobacter cloacae & $23(6)$ & $14(5.5)$ \\
Klebsiella oxytoca & $18(5)$ & $7(2)$ \\
Pseudomonas aeruginosa & $16(4)$ & $15(6)$ \\
Citrobacter freundii & $5(1)$ & $5(2)$ \\
Others GNB & $19(5)$ & $17(6.5)$ \\
ESBL & $38(10)$ & $27(10)$ \\
Gram-positive bacilli & $76(20.5)$ & $81(31)$ \\
Enterococcus faecium & $26(7)$ & $22(8.5)$ \\
Streptococcus spp. & $19(5)$ & $5(2)$ \\
Enterococcus faecalis & $17(4.5)$ & $34(13)$ \\
Staphylococcus aureus & $3(1)$ & $4(1.5)$ \\
Others GP & $11(3)$ & $16(6)$ \\
Anaerobes & $19(5)$ & $9(3.5)$ \\
Clostridium perfringens & $9(2.5)$ & $14(5.5)$ \\
Bacteroides fragilis & $6(1.5)$ & $1(0.5)$ \\
Clostridium difficile & $2(0.5)$ & \\
Others & $2(0.5)$ & \\
Fungi & $2(0.5)$ & \\
Cytomegalovirus & & \\
Cryptosporidium & & \\
\hline GNBGra & & \\
\hline
\end{tabular}

GNB, Gram negative bacteria; ESBL, extended spectrum beta lactamase, GP, Gram positive

data are summarized in Table 3. In blood or bile culture, the most frequently identified bacteria were Gram-negative bacilli (68.5\%) and Gram-positive (24.5\%). Anaerobes $(4.5 \%)$ and fungi $(2.5 \%)$ were relatively uncommon. The most frequently identified bacteria were Escherichia coli (38\%), Klebsiella pneumoniae (9\%), Enterococcus faecalis (8\%), Enterococcus faecium (7.5\%), Enterobacter cloacae (6\%) and Pseudomonas aeruginosa (5\%). Finally, an infection caused by Extended spectrum beta lactamase (ESBL)-producing bacteria was observed in $44 \mathrm{AC}$ patients (12\%). Interestingly, in our cohort the prevalence of ESBL-producing bacteria increases over time $(p=0.011$, Chi-squared for a trend) (Fig. 1). In multivariate analysis, non-gallstone biliary tract obstacle was significantly associated with ESBL bacterial infection (OR 2.13 [1.11-4.21], $p=0.02$ ) (Additional file 3).

\section{Predictive factors of mortality}

Overall in-hospital mortality was $29 \%$. Difference in ACassociated mortality between participating centers could 


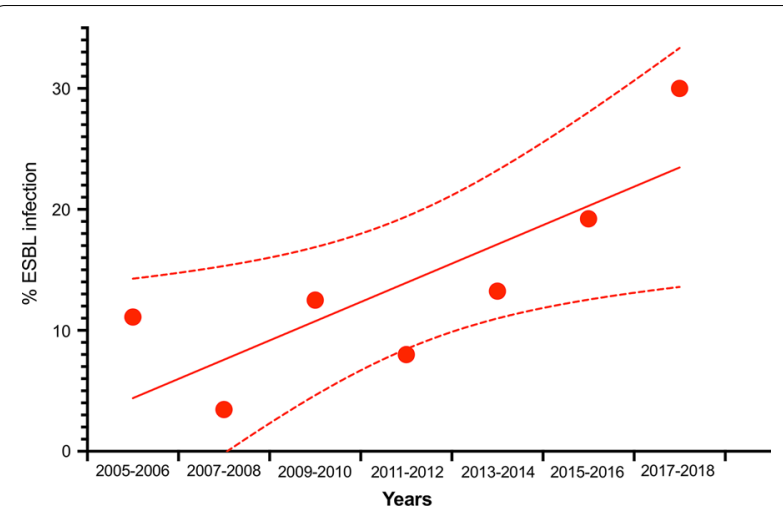

Fig. 1 Observed annual percentage of Extended spectrum beta lactamase producing bacterial infections on documented acute cholangitis in ICUs. The line is a linear regression with 95\% Cls

not be ruled out ( $\mathrm{p}$-value for permutation test $=0.06$ ) (Additional file 4). Several factors were significantly associated with in-hospital death (Fig. 2): SOFA score without bilirubin item, by point (OR 1.15 [95\% CI 1.06-1.25], $p=0.001)$, arterial lactate, by $1 \mathrm{mmol} / \mathrm{L}$ (OR $1.24[95 \% \mathrm{CI}$ $1.10-1.40], p<0.001)$, total serum bilirubin; by $50 \mu \mathrm{mol} / \mathrm{L}$ (OR 1.26 [95\% CI 1.12; 1.42], $p<0.001$ ), tumor as an etiology of AC (OR 2.27 [95\% CI 1.07-4.85], $p=0.01$ ), an initial AC-associated complication (OR 2.74 [95\% CI
1.45-5.17], $p=0.002)$ and an appropriate empirical antibiotic therapy (OR 0.42 [95\% CI $0.20-0.87$ ], $p=0.02$ ). In-hospital mortality significantly decreased overtime, adjusted OR for mortality per year was 0.72 [0.54-0.96] $(p=0.02)$ (Fig. 3).

To analyze the effect of time from admission to biliary drainage on hospital mortality, we performed a landmark analysis adjusted on these factors with 2 predefined time points: 24 and $48 \mathrm{~h}$ after admission (Fig. 4). We found a significant relationship between delayed drainage $(>48 \mathrm{~h})$ and hospital mortality (OR 2.69 [1.18-6.13], $p=0.02$ ). However, no relationship between early drainage $(<24 \mathrm{~h})$ and hospital mortality (OR 1.15 [0.61-2.18], $p=0.66$ ) was found.

\section{Discussion}

Here we describe the characteristics of $382 \mathrm{AC}$ patients admitted in 11 ICUs in France over a 14-year period. In a multivariate regression model, we identified several independent predictive factors of mortality including SOFA score at admission; total bilirubin level, lactate level, non-gallstone-related biliary obstacles and local ACrelated complications. Appropriate empirical antimicrobial therapy was associated with better survival whereas delayed biliary decompression $(>48 \mathrm{~h}$ ) worsened patient outcome.

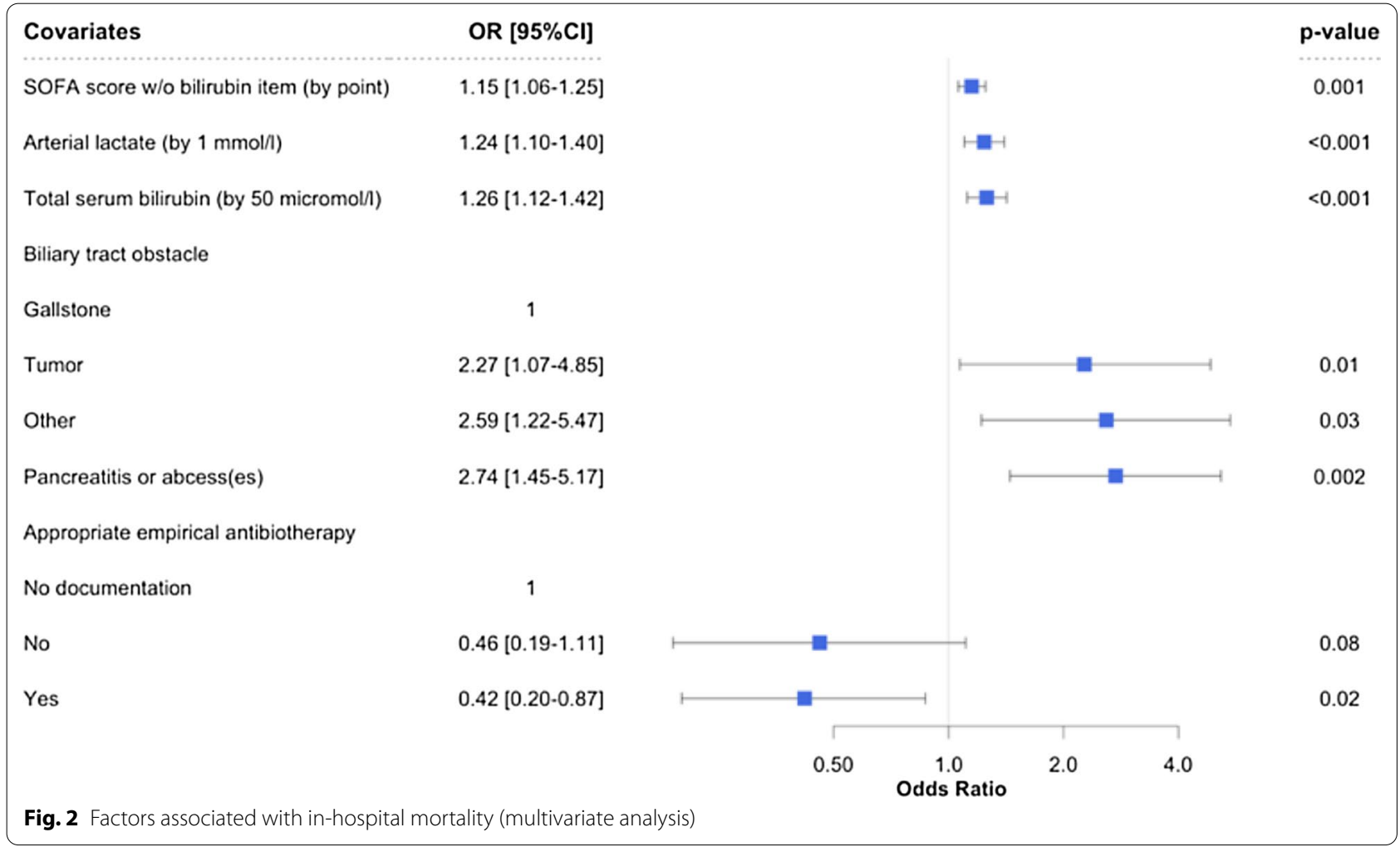




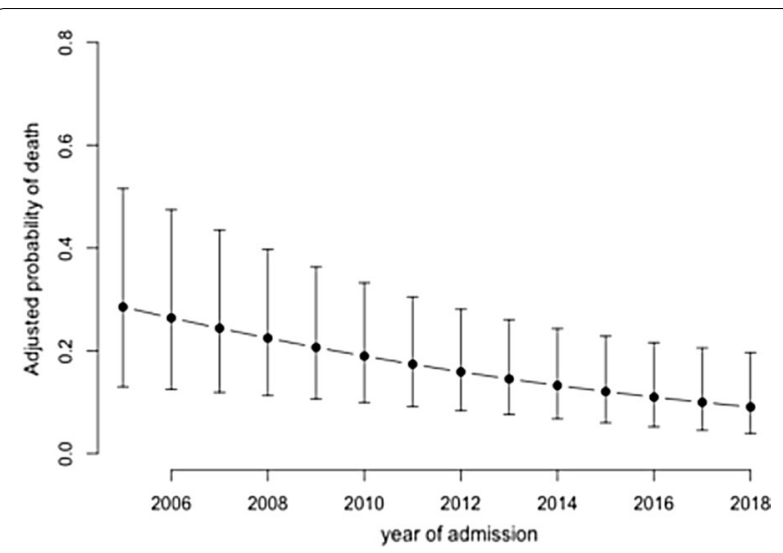

Fig. 3 Adjusted probability of in-hospital mortality across years

The most appropriate time to perform biliary drainage still remains unknown and has to be investigated because not all hospitals have access to ERCP, interventional radiology and hepatobiliary surgery at night or on week-ends. Transferring critically ill patients with AC could be a possibility, but remains challenging in case of uncontrolled acute circulatory failure [11-14]. In our observational "real life" study, we collected data from different regions in France including centers that may or may not have access to $\mathrm{ERCP} /$ interventional radiology. AC-associated mortality was not different between centers and we found that delayed biliary drainage (after $48 \mathrm{~h}$ ) is associated with in-hospital mortality, after adjustment on several confounders including disease severity and appropriate empirical antimicrobial therapy. Previous studies on hospitalized AC patients have reported that delayed time to decompression was associated with longer lengths of hospital stay ( $>24 \mathrm{~h}$ ) [15], prolonged duration of organ failure $(>48 \mathrm{~h})$ [14] or increased hospital readmission $(>48 \mathrm{~h}$ ) [16]. However, none of them addressed the question of when to perform biliary decompression in high-risk AC patients with organ failure. Karvellas et al. found that delaying biliary decompression after $12 \mathrm{~h}$ was associated with increased mortality in patients with septic shock, whereas we identified a cut-off at $48 \mathrm{~h}$. Such discrepancy could be explained by several differences in terms of population, drainage procedure or global mortality. This result highlights that the absence of ERCP availability during the entire week-end is a major issue, especially for patients admitted in ICUs on Fridays. Opening interventional endoscopic centers on Saturdays or Sundays could be an interesting option to improve outcome of critically ill patients with $\mathrm{AC}$ admitted in ICUs at the end of the week. Our results also highlighted that delayed biliary decompression $>24 \mathrm{~h}$ after ICU admission was not associated with higher mortality, suggesting that the first hours of AC patients management should mainly be devoted to resuscitation including antibiotic therapy and organ support therapy (mechanical ventilation, vasopressors, fluids) but not on emergency biliary decompression, especially if the patient has to be transferred to another hospital for ERCP. It is only after hemodynamic optimization, that biliary decompression

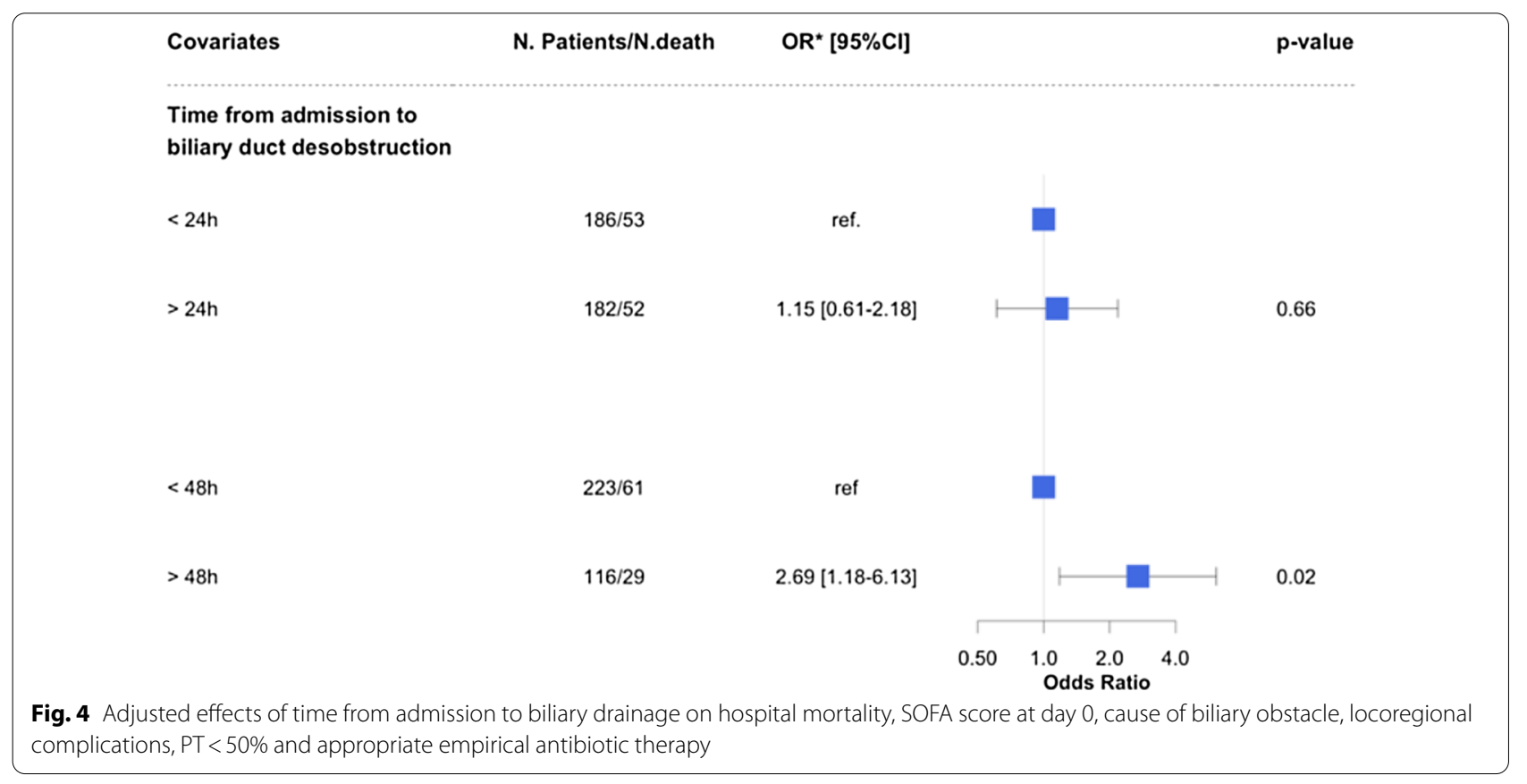


should be considered. Such a conclusion based on retrospective data has to be confirmed prospectively.

In our study, patients were managed according to the International Guidelines with early resuscitation, biliary drainage (87\%) and appropriate empirical antibiotic therapy (75\%). Using multivariate analysis, we found that appropriate empirical antibiotic therapy is a protective factor, corroborating with Karvellas and colleagues' conclusion as well as with studies focusing on peritonitis [17], nosocomial pneumonia [18] and acute bacterial meningitis [19]. First line antibiotic therapy must target gram negative bacteria, mainly Enterobacteriaceae that are frequently found both in blood and bile cultures. Interestingly, identifying ESBL-producing bacteria is not rare. Their prevalence seems to increase over time, suggesting that combining aminoglycoside with a $\beta$-lactam drug to broaden the spectrum should be recommended in critically ill patients with $\mathrm{AC}$, especially with those having non-gallstone obstacles.

Patients had severe ACs with high SOFA scores and frequent use of organ support therapies. In-hospital mortality was $29 \%$ and decreased overtime. Mortality is lower than that reported in Karvellas'study [11]. However, these authors exclusively included patients with $\mathrm{AC}$ and shock whereas in our study, only $61 \%$ of the patients received vasopressors. In addition, strategy for biliary decompression was different, as in their study percutaneous biliary drainage was used as the first-line technique for sepsis source control whereas in our cohort, ERCP was performed in $76 \%$ of the cases. We found that ERCP performed in critically ill patients is safe with low complication rates, most of them being mild bleeding. In addition, ERCP was efficient, considered as optimal in $70 \%$ of the case. Non-optimal procedure may be due to infiltrating tumor-related AC supporting that a non-gallstone biliary obstacle is an independent pejorative prognosis factor $[13,20]$.

Most descriptive studies on AC evaluated hospitalized patients [21-23], and few data is available on critically ill patients admitted in ICUs. Comorbidities and pancreatobiliary malignancies were more frequent in our study, in comparison with Gomi and colleague's study, which included patients from gastroenterology departments [24]. One important take-home message for clinicians is that fever being less frequently observed in our cohort, diagnosis of AC should not be ruled out in the absence of fever. We speculated that low frequency of fever in our cohort could be explained, at least in part, by antibiotic administration before ICU transfer as well as antipyretic use. In our cohort, blood cultures were positive in $68 \%$ of the cases, which is similar to the proportion previously reported on patients with AC and shock. However, it is twofold higher than in Lee and colleague's study [21] which included all hospitalized patients with AC. In the latter study, bacteremia was associated with persistent organ failure at Day 2 [14] whereas in ICUs, positive blood culture was not identified as an independent predictive factor of mortality [25].

Our study should be interpreted in the light of its strengths and limitations. We have included patients from 11 ICUs across several geographic regions in France over a 14-year period, making this study the largest to date on patients with acute cholangitis managed in ICUs. The observed results are thus widely generalizable. Regarding its limitations, this study being an observational retrospective study, only association and not causality can be inferred. Given that this study was observational, we cannot conclusively exclude sources of selection bias. Finally, some data are lacking such as the time between symptom onset and ICU admission and also the causes of delayed biliary decompression.

\section{Conclusion}

In this large multicenter study, we showed that acute cholangitis is a severe condition leading to high mortality in ICU patients. Severity of organ failure, causes of obstruction and local complications of AC are risk factors for mortality, as well as delayed biliary drainage. The impact of biliary decompression timing on mortality has to be prospectively evaluated in future studies.

\section{Supplementary Information}

The online version contains supplementary material available at https://doi. org/10.1186/s13054-021-03480-1.

Additional file 1. Flow chart. Additional file 2. Number of patients with AC included in each center. Additional file 3. Variables associated with EBSL infection. Additional file 4. AC-associated mortality in participating centers. Crude mortality rate by center (left) and distribution of center effects on mortality rate (right) adjusted on individual confounders.

Centers are sorted by study size. Black squares represent adjusted center effects on the mean mortality risk as odds ratio (OR) (comparison of each center to a theoretical average reference center with $\mathrm{OR}=1$ ).

\section{Abbreviations}

AC: Acute cholangitis; ERCP: Endoscopic Retrograde Cholangiopancreatography; ESBL: Extended spectrum beta lactamase; ICU: Intensive care unit; OR: Odds ratio; MAP: Mean arterial pressure; SOFA: Sequential organ failure assessment; SAPS II: Simplified acute physiologic score II.

\section{Acknowledgements}

We are indebted to Rozenn Leboursicaud for her help in data extraction.

\section{Authors' contributions}

Study concept and design, all authors. Acquisitions of data, all authors. Drafting of the manuscript, J.R.L. G.D., H.A.O. Critical revision of manuscript, all authors. Statistical analysis, J.R.L, G.D. and H.A.O. All authors read and approved the final manuscript.

Funding

None. 


\section{Availability of data and materials}

Not applicable.

\section{Ethics approval and consent to participate}

The French Society of Intensive Care ethics committee approved the study (CE SRLF 19-25). Written informed consent was not required, in compliance with French law on retrospective studies of anonymized data. All local medico-administrative data bases are declared to the Commission Nationale Informatique et Liberté (CNIL).

\section{Consent for publication}

Not applicable.

\section{Competing interests}

None.

\section{Author details}

${ }^{1}$ Assistance Publique - Hôpitaux de Paris (AP-HP), Service de médecine intensive et réanimation, Hôpital Saint-Antoine, 184 rue du Faubourg Saint-Antoine, 75571 Paris Cedex 12, France. ${ }^{2}$ Sorbonne Université, Paris, France. ${ }^{3}$ Groupe Hospitalier Sud Île-De-France (GHSIF), Service de réanimation polyvalente, Hôpital de Melun-Sénart, 77000 Melun, France. ${ }^{4}$ Assistance Publique - Hôpitaux de Paris (AP-HP), Service de médecine intensive et réanimation, Hôpital Cochin, 75014 Paris Cedex 12, France. ${ }^{5}$ Centre Hospitalier Argenteuil, Service de réanimation polyvalente et unité de surveillance continue, 95107 Argenteuil, France. ${ }^{6}$ Centre Hospitalier Universitaire Lille, Service de réanimation générale, Hôpital Salengro, 59037 Lille, France. ${ }^{7}$ Service de réanimation polyvalente, Hôpital Privé Claude Galien, 91480 Quincy-sous-Sénart, France. ${ }^{8}$ Service de médecine intensive et réanimation, Centre Hospitalier Universitaire Nantes, Hôtel-Dieu, 44000 Nantes, France. ${ }^{9}$ Centre Hospitalier Intercommunal Poissy Saint-Germain-en-Laye, Service de réanimation, Hôpital de Poissy, 78303 Poissy, France. ${ }^{10}$ Centre Hospitalier Régional Universitaire Besançon, Service de réanimation médicale, Hôpital Jean Minjoz, 25030 Besançon, France. ${ }^{11}$ Centre Hospitalier de Saint-Denis, Service de réanimation et soins continus, Hôpital Delafontaine, 93205 Saint-Denis, France. ${ }^{12}$ Centre Hospitalier Sud Seine-et-Marne, Service de réanimation, Hôpital Fontainebleau, 77300 Fontainebleau, France. ${ }^{13}$ Inserm U970, Centre de Recherche Cardiovasculaire de Paris (PARCC), Paris, France. ${ }^{14}$ Assistance Publique - Hôpitaux de Paris (AP-HP), Centre d'endoscopie digestive, Hôpital Saint-Antoine, Paris, France.

Received: 13 November 2020 Accepted: 25 January 2021

Published online: 06 February 2021

\section{References}

1. Kiriyama S, Kozaka K, Takada T, Strasberg SM, Pitt HA, Gabata T, et al. Tokyo Guidelines 2018: diagnostic criteria and severity grading of acute cholangitis (with videos). J Hepatobiliary Pancreat Sci. 2018;25:17-30.

2. Leligdowicz A, Dodek PM, Norena M, Wong H, Kumar A, Kumar A. Association between source of infection and hospital mortality in patients who have septic shock. Am J Respir Crit Care Med. 2014;189:1204-13.

3. Baykara N, Akalın H, Arslantaş MK, Hancı V, Çağlayan Ç, Kahveci F, et al. Epidemiology of sepsis in intensive care units in Turkey: a multicenter point-prevalence study. Crit Care. 2018;22:93.

4. Annane D, Aegerter P, Jars-Guincestre MC, Guidet B. Current epidemiology of septic shock: the CUB-Réa network. Am J Respir Crit Care Med. 2003;168:165-72.

5. SepNet Critical Care Trials Group. Incidence of severe sepsis and septic shock in German intensive care units: the prospective, multicentre INSEP study. Intensive Care Med. 2016;42:1980-9.

6. Miura F, Okamoto K, Takada T, Strasberg SM, Asbun HJ, Pitt HA, et al. Tokyo Guidelines 2018: initial management of acute biliary infection and flowchart for acute cholangitis. J Hepato-Biliary-Pancreatic Sci. 2018;25:31-40.

7. Solomkin JS, Mazuski JE, Bradley JS, Rodvold KA, Goldstein EJC, Baron EJ, et al. Diagnosis and management of complicated intra-abdominal infection in adults and children: guidelines by the surgical infection society and the infectious diseases society of America. Clin Infect Dis. 2010;50:133-64.

8. Singer M, Deutschman CS, Seymour CW, Shankar-Hari M, Annane D, Bauer $M$, et al. The third international consensus definitions for sepsis and septic shock (Sepsis-3). JAMA. 2016;315:801.

9. Leclercq R, Cantón R, Brown DFJ, Giske CG, Heisig P, MacGowan AP, et al. EUCAST expert rules in antimicrobial susceptibility testing. Clin Microbiol Infect. 2013;19:141-60.

10. Dumas G, Demoule A, Mokart D, Lemiale V, Nseir S, Argaud L, et al. Center effect in intubation risk in critically ill immunocompromised patients with acute hypoxemic respiratory failure. Crit Care. 2019;23:306.

11. Karvellas CJ, Abraldes JG, Zepeda-Gomez S, Moffat DC, Mirzanejad Y, Vazquez-Grande G, et al. The impact of delayed biliary decompression and anti-microbial therapy in 260 patients with cholangitis-associated septic shock. Aliment Pharmacol Ther. 2016;44:755-66.

12. Lai EC, Mok FP, Tan ES, Lo CM, Fan ST, You KT, et al. Endoscopic biliary drainage for severe acute cholangitis. N Engl J Med. 1992;326:1582-6.

13. Khashab MA, Tariq A, Tariq U, Kim K, Ponor L, Lennon AM, et al. Delayed and unsuccessful endoscopic retrograde cholangiopancreatography are associated with worse outcomes in patients with acute cholangitis. Clin Gastroenterol Hepatol. 2012;10:1157-61.

14. Lee F, Ohanian E, Rheem J, Laine L, Che K, Kim JJ. Delayed endoscopic retrograde cholangiopancreatography is associated with persistent organ failure in hospitalised patients with acute cholangitis. Aliment Pharmacol Ther. 2015;42:212-20.

15. Jang SE, Park SW, Lee BS, Shin CM, Lee SH, Kim J-W, et al. Management for CBD stone-related mild to moderate acute cholangitis: urgent versus elective ERCP. Dig Dis Sci. 2013;58:2082-7.

16. Navaneethan U, Gutierrez NG, Jegadeesan R, Venkatesh PGK, Butt M, Sanaka MR, et al. Delay in performing ERCP and adverse events increase the 30 -day readmission risk in patients with acute cholangitis. Gastrointest Endosc. 2013;78:81-90

17. On behalf of the OUTCOMEREA study group, Morvan A-C, Hengy B, Garrouste-Orgeas M, Ruckly S, Forel J-M, et al. Impact of species and antibiotic therapy of enterococcal peritonitis on 30-day mortality in critical care-an analysis of the OUTCOMEREA database. Crit Care. 2019;23:307.

18. Iregui M, Ward S, Sherman G, Fraser VJ, Kollef MH. Clinical Importance of delays in the initiation of appropriate antibiotic treatment for ventilatorassociated pneumonia. Chest. 2002;122:262-8

19. Proulx N, Frechette D, Toye B, Chan J, Kravcik S. Delays in the administration of antibiotics are associated with mortality from adult acute bacterial meningitis. OJM. 2005:98:291-8.

20. Aboelsoud M, Siddique O, Morales A, Seol Y, Al-Qadi M. Early biliary drainage is associated with favourable outcomes in critically-ill patients with acute cholangitis. Gastroenterol Rev. 2018;13:16-21.

21. Lee C-C, Chang I-J, Lai Y-C, Chen S-Y, Chen S-C. Epidemiology and prognostic determinants of patients with bacteremic cholecystitis or cholangitis. Am J Gastroenterol. 2007;102:563-9.

22. Kiriyama S, Takada T, Hwang T-L, Akazawa K, Miura F, Gomi H, et al. Clinical application and verification of the TG13 diagnostic and severity grading criteria for acute cholangitis: an international multicenter observational study. J Hepato-Biliary-Pancreatic Sci. 2017;24:329-37.

23. Tan M, Schaffalitzky-de-Muckadell OB, Laursen SB. Association between early ERCP and mortality in patients with acute cholangitis. Gastrointest Endosc. 2018;87:185-92.

24. Gomi H, Takada T, Hwang T-L, Akazawa K, Mori R, Endo I, et al. Updated comprehensive epidemiology, microbiology, and outcomes among patients with acute cholangitis. J Hepato-Biliary-Pancreatic Sci. 2017;24:310-8.

25. Amaro R, Liapikou A, Cilloniz C, Gabarrus A, Marco F, Sellares J, et al. Predictive and prognostic factors in patients with blood-culturepositive community-acquired pneumococcal pneumonia. Eur Respir J. 2016;48:797-807.

\section{Publisher's Note}

Springer Nature remains neutral with regard to jurisdictional claims in published maps and institutional affiliations. 